\title{
Radiological staging in breast cancer: which asymptomatic patients to image and how
}

\author{
T Barrett', DJ Bowden', DC Greenberg' ${ }^{2}$ CH Brown', GC Wishart ${ }^{3,4}$ and PD Britton ${ }^{*, 3,4}$ \\ 'Department of Radiology, Box 219, Addenbrooke's Hospital, Cambridge University Teaching Hospitals NHS Foundation Trust, Hills Road, Cambridge \\ CB2 OQQ UK: ${ }^{2}$ Eastern Cancer Registration and Information Centre, Shelford Bottom, Cambridge CB22 3AD, UK; ${ }^{3}$ Cambridge Breast Unit, Box 97 , \\ Cambridge University Hospitals NHS Foundation Trust, Hills Road, Cambridge CB2 OQQ, UK; ${ }^{4}$ Cambridge Biomedical Research Centre, National Institute \\ of Health Research, Hills Road, Cambridge CB2 OQQ, UK
}

BACKGROUND: Approximately 4\% of patients diagnosed with early breast cancer have occult metastases at presentation. Current national and international guidelines lack consensus on whom to image and how.

METHODS: We assessed practice in baseline radiological staging against local guidelines for asymptomatic newly diagnosed breast cancer patients presenting to the Cambridge Breast Unit over a 9-year period.

RESULTS: A total of 2612 patients were eligible for analysis; $91.7 \%$ were appropriately investigated. However in the subset of lymph node negative stage II patients, only 269 out of 354 (76.0\%) investigations were appropriate. No patients with stage 0 or I disease had metastases; only two patients (0.3\%) with stage II and $\leqslant 3$ positive lymph nodes had metastases. Conversely, 2.2, 2.6 and $3.8 \%$ of these groups had false-positive results. The incidence of occult metastases increased by stage, being present in 6, I3.9 and $57 \%$ of patients with stage II ( $\geqslant 4$ positive lymph nodes), III and IV disease, respectively.

CONCLUSION: These results prompted us to propose new local guidelines for staging asymptomatic breast cancer patients: only clinical stage III or IV patients require baseline investigation. The high specificity and convenience of computed tomography (chest, abdomen and pelvis) led us to recommend this as the investigation of choice in breast cancer patients requiring radiological staging.

British Journal of Cancer (2009) I 0I, I522- I528. doi: I0.1038/sj.bjc.6605323 www.bjcancer.com

Published online 29 September 2009

(C) 2009 Cancer Research UK

Keywords: radiological staging; asymptomatic; breast cancer; CT

Breast cancer is second only to lung cancer in worldwide prevalence. It represents $16 \%$ of all UK cancers and is the most common cancer in women, accounting for $31 \%$ of all cancers (WHO, 2003, 2006). The incidence of breast cancer amongst women in the UK was 45660 in 2005 (ONS, 2008). Approximately $4 \%$ of breast cancer patients will have detectable metastatic disease at the time of diagnosis; the majority of these patients will have signs or symptoms of metastases (Ravaioli et al, 2002). The most common first sites of distant metastases are bone, lung, brain and liver; up to $10 \%$ of patients with metastatic disease will have lesions at multiple sites (Patanaphan et al, 1988). Conventionally, high-risk patients have been screened for occult metastases in these organs by using chest radiographs (CXR), liver ultrasound (US) and bone scintigraphy (BS) (Puglisi et al, 2007). More recently, techniques such as computed tomography (CT), magnetic resonance imaging and ${ }^{18} \mathrm{FDG}$-positron emission tomography (Avril et al, 2007) have started to be used in certain circumstances, or for problem-solving.

The likelihood of detecting metastatic disease at the time of initial diagnosis is highly dependant upon the extent of locoregional disease and the presence of symptoms. The detection of

* Correspondence: Dr PD Britton;

E-mail: peter.britton@addenbrookes.nhs.uk

Received 3 June 2009; revised 14 August 2009; accepted 21 August 2009; published online 29 September 2009 such metastases has a profound effect upon patient management and prognosis, however, there is little evidence to guide decisions on which patients should be staged. Where symptoms are suggestive of metastatic disease, appropriate investigations targeted to the respective organs should be instigated. However, the vast majority of newly diagnosed patients do not exhibit symptoms or signs of metastatic disease. As metastatic rates overall are so low, staging all patients would result in unacceptable expenditure, increased workload, patient anxiety and radiation burden. Imaging staging of patients needs to be targeted towards those most likely to have disseminated disease. The aim of this paper was to assess practice in baseline radiological staging against local guidelines of a large cohort of breast cancer patients stratified by clinico-pathological stage and additionally determine the likelihood of detecting metastases and, as importantly, obtaining a falsepositive result. We also aimed to explore the current trend to use CT rather than conventional assessment with CXR, US liver and BS.

\section{MATERIALS AND METHODS}

A list of all female patients diagnosed with breast cancer during a 9-year period, between 1 January 1999 and 31 December 2007, was obtained from the Eastern Cancer Registration and Information Centre (ECRIC) database. Each patient was prospectively allocated a clinico-pathological staging classification by the ECRIC medical director and clinical oncologist (author $\mathrm{CHB}$ ). The classification 
Table I Assessed patient numbers

\begin{tabular}{|c|c|c|c|c|c|c|}
\hline Stage & $\begin{array}{c}\text { Total } \\
\text { cases } \\
\text { | 999-2007 }\end{array}$ & $\begin{array}{c}\text { Total } \\
\text { (\%) }\end{array}$ & $\begin{array}{c}\text { Patients } \\
\text { meeting } \\
\text { inclusion } \\
\text { criteria }\end{array}$ & $\begin{array}{c}\text { Total } \\
\text { (\%) }\end{array}$ & $\begin{array}{c}\text { NCDB } \\
\text { case } \\
\text { data }^{a}\end{array}$ & $\begin{array}{c}\text { NCDB \% } \\
\operatorname{data}^{a}\end{array}$ \\
\hline 0 & 432 & 12.7 & 348 & 13.3 & 57796 & 17.1 \\
\hline I & 1155 & 34 & 992 & 38 & $|40| 22$ & 41.4 \\
\hline$\|$ & 1388 & 40.8 & $104 \mid$ & 39.8 & 106116 & 31.3 \\
\hline III & 244 & 7.2 & 224 & 8.6 & 22758 & 6.7 \\
\hline IV & 145 & 4.3 & 7 & 0.3 & 11780 & 3.5 \\
\hline Unknown & 34 & 1 & 0 & 0 & 0 & 0 \\
\hline Overall & 3398 & 100 & 2612 & 100 & 338572 & 100 \\
\hline
\end{tabular}

Abbreviation: $\mathrm{NCDB}=$ National Cancer Data Base. Inclusion criteria: female patients with a new diagnosis of breast cancer established between I January 1999 and 31 December 2007. Exclusion criteria: diagnosis not established at Addenbrooke's, or accurate stage could not be established - see text for full explanation. Reports, vl.I . Chicago, IL, 2002. http://www.facs.org/cancer/ncdb/publicaccess.html. ${ }^{a}$ Comparative figures taken from the National Cancer Data Base, USA 1998 - 1999: cases diagnosed in 1247 US nationwide hospitals in 1998 and 1999: Commission on Cancer, American College of Surgeons. NCDB Benchmark.

was undertaken 6 months following initial diagnosis by reviewing the medical records and was based on clinical, imaging and pathological data. The overall distribution of patients between stages is comparable to data published by the National Cancer Data Base, USA (Table 1).

Staging was allocated according to the TNM (tumour, node, metastasis) staging system (0-IV) published in the American Joint Committee on Cancer (AJCC) staging manual. The AJCC sixth edition was published in 2002 (Greene et al, 2002), replacing the fifth edition from 1997 (Fleming et al, 1997) and contained some changes particularly relevant to our study. In the sixth edition ' $\mathrm{N}$ stages' were changed to give greater emphasis to the number of nodes involved: 4-9 nodes (or any internal mammary lymph nodes) became $\mathrm{N} 2$ disease, and $\geqslant 10$ axillary nodes now qualified as $\mathrm{N} 3$ disease. $\mathrm{N} 1$ disease classifies the patient as stage II, whereas N2 or N3 disease places a patient in the stage III category (Supplementary Table 1). For example, patients with four positive axillary lymph nodes would be stage IIb using the criteria of the fifth edition, but stage IIIa under the sixth edition. To avoid confusion, we retained the criteria used in the fifth edition, but subdivided our results for stage II disease into patients with three nodes or less (stage II-i) or more than four nodes (stage II-ii) involved. It should be noted that patients with $\mathrm{N} 1$ status can still have stage III or IV disease, if the tumour is $>5 \mathrm{~cm}$ diameter, or involves the skin or chest wall.

Patients in whom an accurate stage could not be defined due to insufficient information and patients with symptoms, signs or abnormal blood work indicative of metastatic disease were excluded from the data analysis. The Cambridge Breast Unit is a tertiary referral centre and treats some patients initially diagnosed with breast cancer at another centre. As an accurate investigation history could not always be reliably obtained, patients that did not receive their primary diagnosis of breast cancer at this institution were also excluded. The final exclusion criteria included patients whose breast cancer was diagnosed on the basis of co-incidental abnormal imaging results that suggested metastases and the search for a primary cancer ensued.

With clinical stage being assigned retrospectively, 6 months after diagnosis, patients where imaging had shown metastases were classified as stage IV, however these patients may have initially presented with a lower stage of disease clinically. Thus all stage IV cases were reviewed on an individual basis and patients were re-assigned to the relevant lower stage group based on the information that would have been available at presentation.

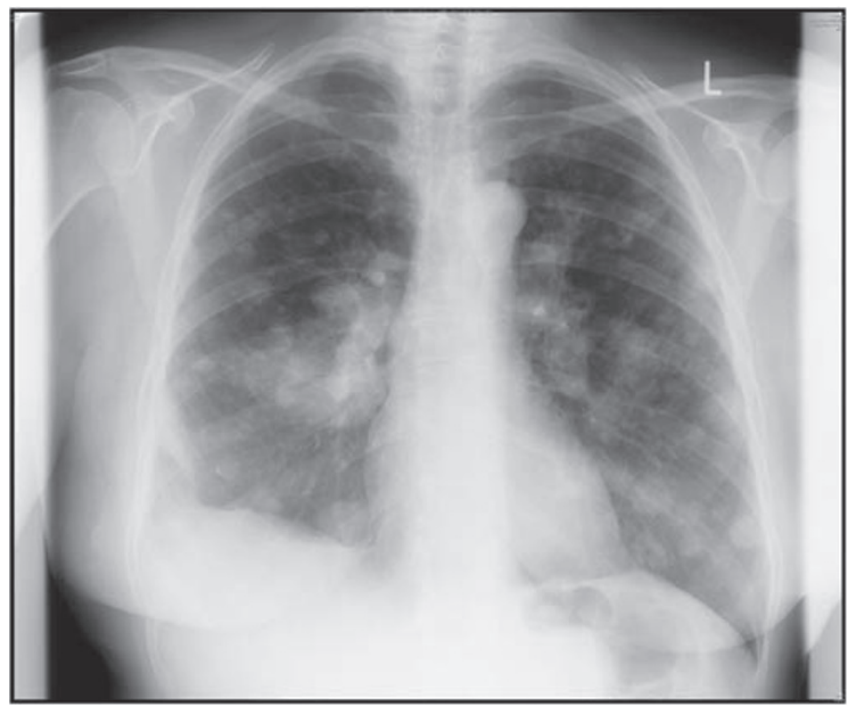

Figure I An 'unequivocal' true positive. Chest $X$-ray showing multiple metastases throughout both lungs in a patient with stage IV breast cancer.

It should be noted that patients can still present with clinical stage IV disease, for example inflammatory breast cancer, thus seven patients remained in this group following the application of exclusion criteria and stage re-assignment.

Baseline staging investigations were arbitrarily chosen to be those taking place within 3 months of the date of breast cancer diagnosis. This was considered to be a reasonable time period for management to be discussed in multi-disciplinary meetings, surgery to take place, and histopathological results to be available and subsequent imaging tests to be performed. Patients were analysed on a case-by-case basis, with imaging investigations, indications and results logged. A true-positive result was determined as being one that unequivocally confirmed metastatic disease on the basis of the respective imaging investigation (Figure 1), or one that was proven by subsequent imaging or histology (Figures 2 and 3). A false-positive result was taken as being any staging investigation that was initially reported as being either abnormal or indeterminate, but upon subsequent investigation(s) was proven to be negative (Figure 4).

The local staging guidelines at the Cambridge Breast Unit (updated in 1999) used as the 'gold standard' for our assessment are as follows:

- Pre-operative CXR is not necessary as a staging investigation unless past medical history warrants, or symptoms warrant or there is a high risk of an ITU admission (reference Making the Best Use of a Department of Clinical Radiology, Royal College of Radiologists)

- Patients with T4 tumours or malignant lymphadenopathy should be considered for pre-operative staging with CXR, liver US and bone scan

- Patients with more locally advanced breast cancer should have investigations directed at findings underlying asymptomatic metastases where this may influence management

Thus, patients with positive lymph nodes or T4 tumours (involvement of chest wall or skin) are eligible for baseline staging investigations according to local guidelines. This includes all tumours that are $\mathrm{N} 1-3$ and/or T4 and any request for CXR, bone scan, US or CT in such patients was considered appropriate. However, staging was not deemed appropriate in stage 0, I and stage II lymph node negative patients; a CXR for pre-operative assessment was considered appropriate if indicated under the RCR guidelines (RCR, 2007). 


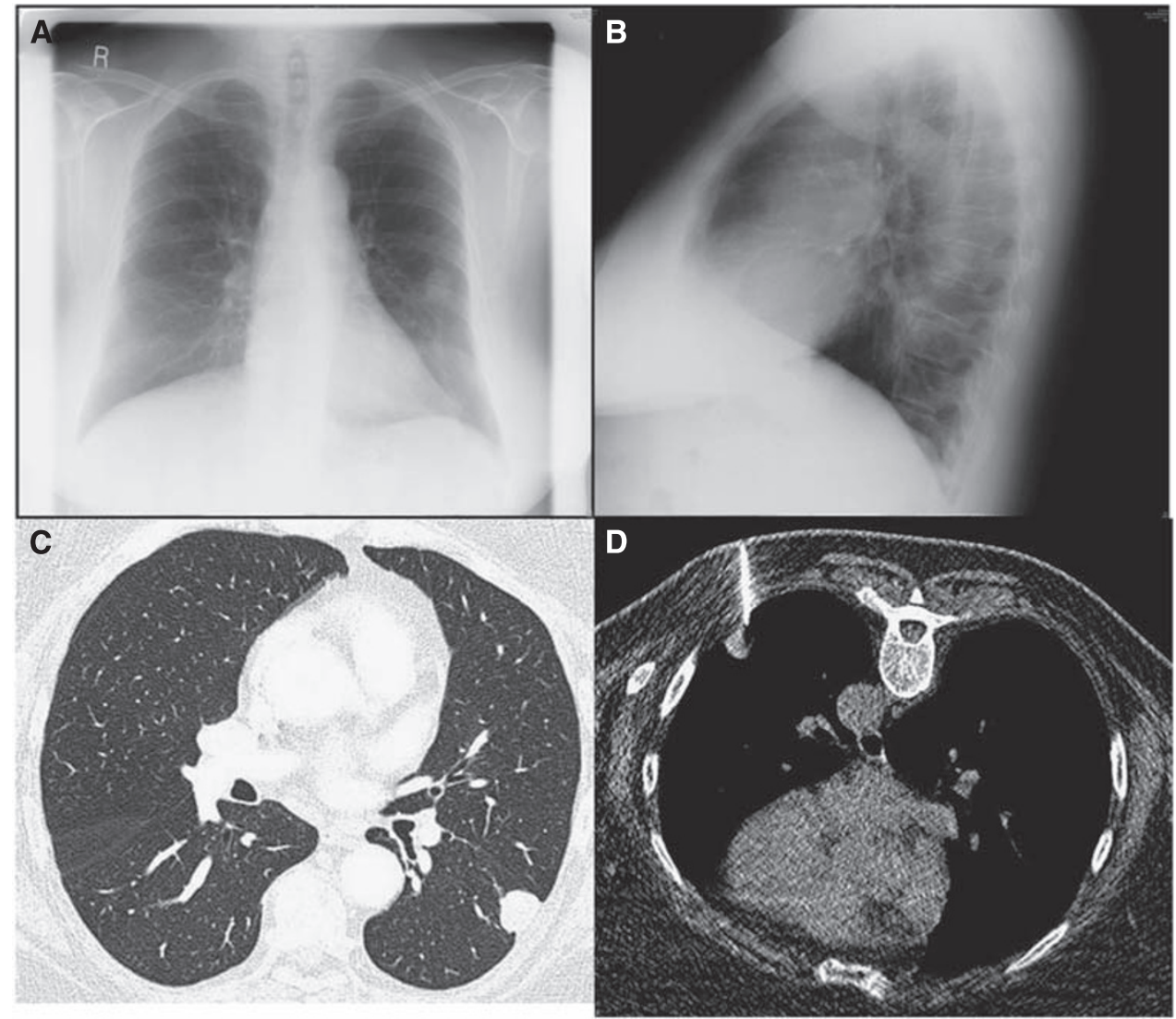

Figure 2 True positive. Chest $X$-ray reveals a 'coin' lesion in the left mid-zone $(\mathbf{A})$, also demonstrated on the lateral radiograph $(\mathbf{B})$. CT examination $(\mathbf{C})$ on lung-window settings supports the diagnosis of a likely lung metastasis, later confirmed by CT-guided biopsy, performed with the patient lying prone (D).

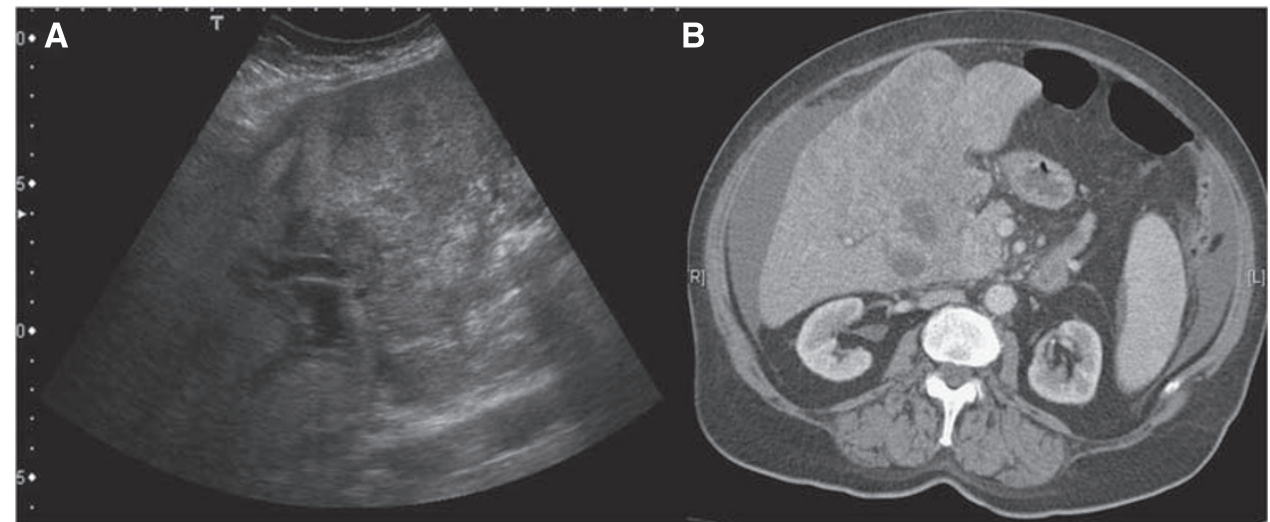

Figure 3 True positive. Stage IV breast cancer patient. Ultrasound liver shows irregular lesions within the liver (A), subsequent CT (B) confirms liver metastases, ascites also demonstrated.

Finally we estimated the health-care costs of detecting metastases by stage of disease and mode of imaging staging. This was based on local costing taking into consideration staffing, consumable and hardware expenses. From the true-positive rates achieved in this study we have calculated how much would need to be spent to detect a single patient with metastatic disease for each disease stage. These estimates have been made for staging by both standard (CXR $£ 80$, US liver $£ 176$ and BS £184) methods and for $\mathrm{CT}$ alone (chest, abdomen, pelvis £271). We have also included in all these estimates the cost of additional imaging generated by the false-positive results in this study.

\section{RESULTS}

Of 3398 patients with newly diagnosed breast cancer over the 9-year study period, 2612 cases were eligible for data analysis (Table 1). Appropriate investigations undertaken in stages $0, \mathrm{I}$, and II-i (stage II with $\leqslant 3$ lymph nodes), that is patients who should receive only a CXR (if clinically indicated) and no additional staging investigations according to local guidelines, were as follows: 325 out of 348 (93.4\%) for stage 0,881 out of 992 for stage I $(88.8 \%)$ and 779 out of 859 for stage II-i (90.7\%). For the subgroup of stage II-i with no lymph nodes involved only 269 out 


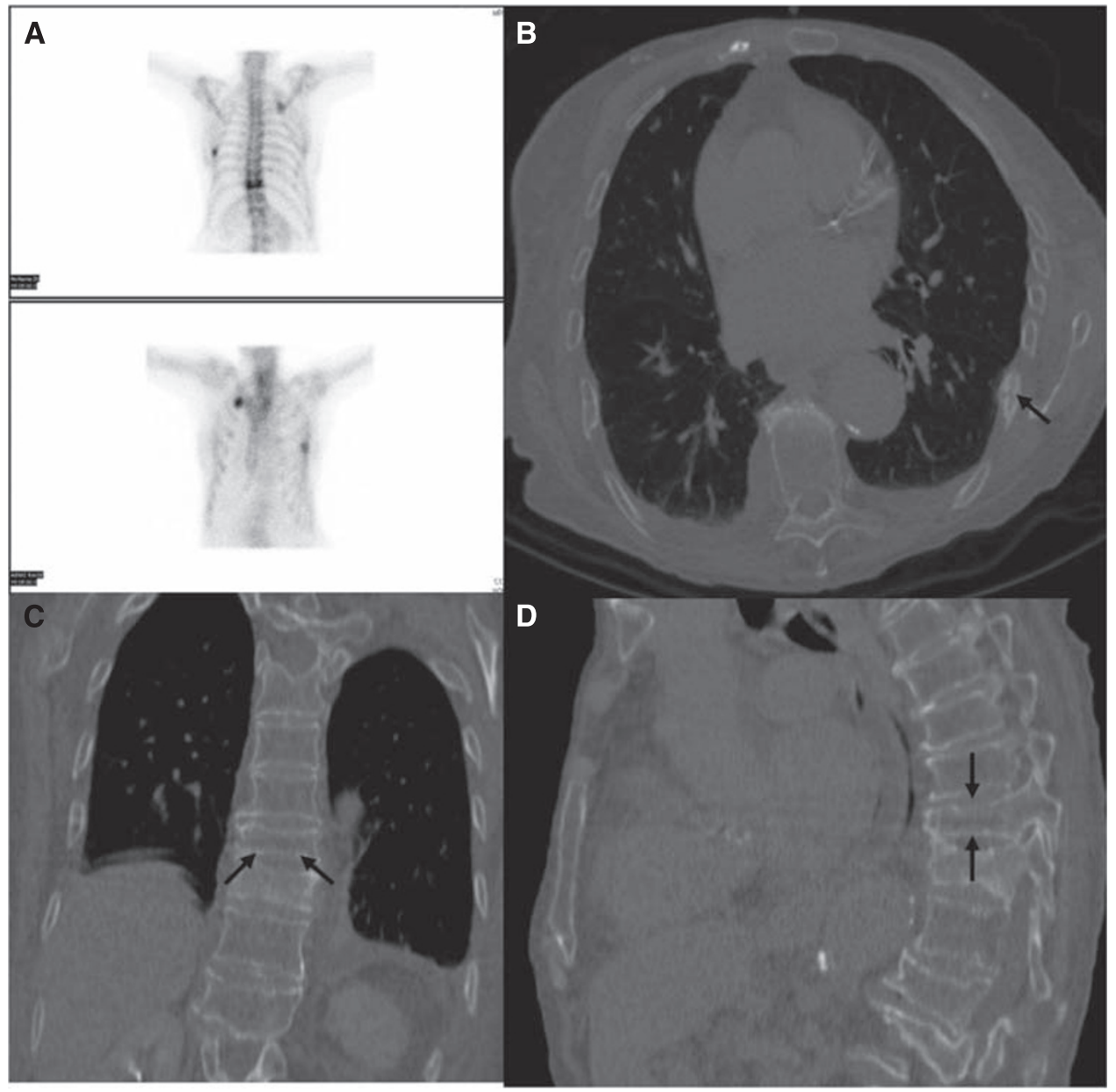

Figure 4 False positive. Bone scintigram $(\mathbf{A})$ and CT chest examination $(\mathbf{B}, \mathbf{C}, \mathbf{D})$ in a patient with stage IV breast cancer. The bone scan was reported as showing hot spots in the TIO vertebrae, left posterior seventh and right anterior second ribs, consistent with metastases. The follow-up CT scan revealed these changes to be due to rib fractures $(\mathbf{B})$, and degenerative disease $(\mathbf{C}, \mathbf{D})$ only.

of 354 requests $(76.0 \%)$ were appropriate. Overall for stages 0 , I and II-i, 1985 out of 2199 (90.3\%) patients were appropriately investigated. Including the 413 patients with stages II-ii, III and IV disease, who were all appropriately investigated, the overall compliance with local guidelines for baseline screening in asymptomatic breast cancer patients was 2398 out of 2612 (91.8\%).

Of the 1340 patients with stages 0 and I (674 of whom had a least one investigation) there were no true-positive results but 17 patients had false-positive results. Of the 859 patients with stage II-i disease there were only 2 potential true-positive results $(0.3 \%$ of cases). The majority (607) of these patients had at least one investigation, and there were $23(2.7 \%)$ false-positive results in this group. There were $10(6 \%)$ true-positive results and $22(13.1 \%)$ false-positive results in patients with stage II-ii; $26(13.9 \%)$ true positives and $24(12.8 \%)$ false positives in stage III and $4(57 \%)$ true positives, with no false-positive results in patients with stage IV disease (Table 2).

The two potential 'true'-positive results in stage II-i patients require further explanation. The first patient was 87 years old at diagnosis and presented with a $22 \mathrm{~mm}$, node negative, grade 2 tumour. A soft tissue lesion was seen in the right lower zone on baseline CXR and reported as representing a metastasis and therefore classified as a true positive in this study. However, 6 years after initial presentation, a repeat CXR showed that the lesion had not changed in size thus casting doubt over the initial malignant diagnosis (Figure 5). In the interest of consistency we have retained the initial classification for our analysis, however it
Table 2 Number of investigations performed by modality and stage of disease and true- and false-positive results by stage of disease

\begin{tabular}{lccccccc}
\hline Stage & $\mathbf{0}$ & $\mathbf{I}$ & $\mathbf{I I - i}$ & $\mathbf{I I - i i}$ & $\mathbf{I I I}$ & $\mathbf{I V}$ & Total \\
\hline Total no. of patients & 348 & 992 & 859 & 182 & 224 & 7 & 2612 \\
Staging investigations & & & & & & & \\
CXR & 136 & 527 & 580 & 148 & 160 & 5 & 1556 \\
US & 0 & 19 & 101 & 100 & 114 & 5 & 339 \\
BS & 0 & 22 & 109 & 113 & 125 & 4 & 373 \\
CT & 3 & 6 & 19 & 15 & 34 & 1 & 78 \\
Total no. of patients having staging investigations & & & & \\
True +ve (\%) & $0(0)$ & $0(0)$ & $2(0.3)$ & $10(6)$ & $26(13.9)$ & $4(57)$ \\
False +ve (\%) & $3(2.2)$ & $14(2.6)$ & $23(3.8)$ & $22(13.1)$ & $24(12.8)$ & $0(0)$ & \\
\hline
\end{tabular}

Abbreviations: $\mathrm{BS}=$ bone scintigraphy; $\mathrm{CT}=$ computed tomography; $\mathrm{CXR}=$ chest radiograph; US = ultrasound. Stage $I I-i=$ patients with stage II disease by AJCC sixth edition (Greene et al, 2002): $\leqslant 3$ lymph nodes positive, stage II-ii= patients with stage II disease by AJCC fifth edition (Fleming et al, 1997), but stage III disease by AJCC sixth edition, i.e. $\geqslant 4$ positive lymph nodes.

is possible that this represents a false- rather than true-positive case. The second true-positive patient in the stage II-i group was 62 years old at diagnosis, and presented with a $38 \mathrm{~mm}$, node negative, grade 3 tumour. Chest radiographs suggested lung metastases, subsequently confirmed at CT, which additionally showed 

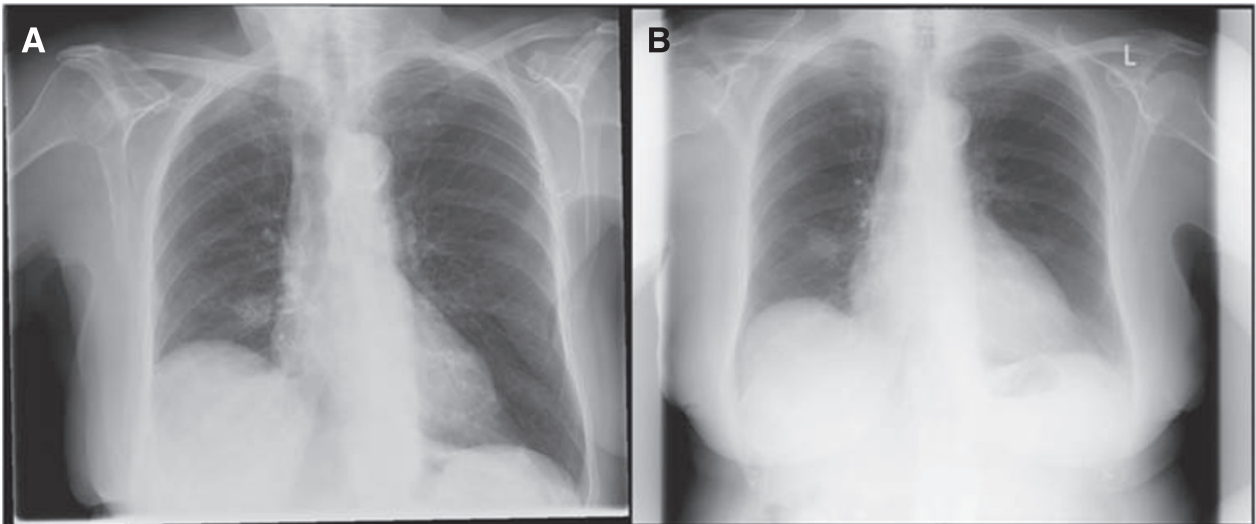

Figure 5 One of two patients with stage II-i disease with a 'true-positive' diagnosis. The soft tissue lesion in the right lower zone was reported as being a metastatic lesion on baseline CXR shortly after the patient presented with primary breast cancer in 200I (A). However, in 2007 the lesion had not significantly changed in size (B) and was retrospectively described as being 'unlikely to be malignant'.

Table 3 True- and false-positive results by modality (includes all stages of disease)

\begin{tabular}{lcccc}
\hline & CXR & US & BS & CT \\
\hline Total & 1556 & 339 & 373 & 78 \\
True +ve (\%) & $3(0.2)$ & $6(1.8)$ & $23(6.2)$ & $21(26.9)$ \\
False +ve (\%) & $20(1.3)$ & $13(3.8)$ & $51(13.7)$ & $3(3.8)$ \\
\hline
\end{tabular}

Abbreviations: $\mathrm{BS}=$ bone scintigraphy; $\mathrm{CT}=$ computed tomography; $\mathrm{CXR}=$ chest radiograph; US = ultrasound

peritoneal metastases. The patient was a smoker with a history of hypertension, thus met local guidelines for a pre-operative CXR. A subsequent clinic letter documented erythema and thickening of the axilla; skin involvement would make the tumour T4 (stage IIIb), thus it is possible that this case represents an error in stage assignment.

There was a difference in the accuracy of the four different imaging modalities used. There were 3 true-positive $(0.2 \%)$ and 20 (1.3\%) false-positive results out of 1556 CXRs, 6 true-positive $(1.8 \%)$ and $13(3.8 \%)$ false-positive results out of 339 liver US, 23 true-positive $(6.2 \%)$ and $51(13.7 \%)$ false-positive results out of 373 bone scans, and 21 true-positive $(26.9 \%)$ and $3(3.8 \%)$ falsepositive results out of 78 CTs (Table 3 ). Computed tomography was the only modality in which the percentage of true-positive results was higher than the false-positive results. Computed tomography also had a lower rate of false-positive results $(3.8 \%)$ than US and BS. Of the three false-positive CT results two described small pulmonary nodules of indeterminate significance, which were unchanged on follow-up CT, and one described a liver lesion that was too small to characterise, but was shown to be a cyst on subsequent US. There was a noticeable trend during the study period to request more CT scans, with a corresponding reduction in requests for CXR, US and bone scans.

In this review there were 23 patients with true-positive bone scan results; of these every case had at least one metastasis in the field that would be covered by CT of the chest/abdomen/pelvis. Retrospective analysis of all bone scan results (including some symptomatic patients who were later excluded) revealed that of 411 scans performed, 257 were normal, 41 showed degenerative change, 52 were true-positive results and 61 were false-positive results. In the 52 cases where the bone scan was found to be a true positive, all patients had at least one metastasis within the field that would be covered by CT of the chest, abdomen and pelvis.

The estimated health-care cost of detecting metastases by stage of disease and mode of imaging staging is shown in Table 4. Calculations were carried out on the true-positive rates and the added expense generated by false-positive imaging results. The
Table 4 Estimate of health-care costs to detect metastases by stage of disease and by mode of imaging

\begin{tabular}{lccccccc}
\hline & \multicolumn{7}{c}{ TNM stage } \\
\cline { 2 - 7 } & 0 & I & II-i & II-ii & III & IV \\
\hline $\begin{array}{l}\text { True-positive rate } \\
\text { for detection of } \\
\text { metastases (\%) }\end{array}$ & 0 & 0 & 0.2 & 5.5 & II.6 & 57.0 \\
$\begin{array}{l}\text { Estimated cost of detecting I patient with metastatic disease by } \\
\text { 'Standard' staging }\end{array}$ & Infinite & Infinite & $£ 200393$ & $£ 8492$ & $£ 4021$ & $£ 8$ I7 \\
$\begin{array}{l}\text { Investigations } \\
\text { (CXR, US, BS) } \\
\begin{array}{l}\text { CT staging alone } \\
\hline\end{array}\end{array}$ & Infinite & Infinite & $£ 119744$ & $£ 5074$ & $£ 2405$ & $£ 488$
\end{tabular}

Abbreviations: $\mathrm{BS}=$ bone scintigraphy; $\mathrm{CT}=$ computed tomography; $\mathrm{CXR}=$ chest radiograph; TNM = tumour, node, metastasis; US =ultrasound. Estimates for detecting a single patient with metastatic disease for each disease stage based on local costings and the respective true- and false-positive rates by modality (see text for costing estimates).

CXR, BS and liver US false-positive rates in this study were 1.3, 13.7 and $3.8 \%$ respectively. Every false-positive CXR, BS and US cost $£ 89, £ 132$ and $£ 190$, respectively, in additional imaging investigations. The CT false-positive rate was $3.8 \%$ and every falsepositive CT costs $£ 203$ in additional imaging investigations. As no metastatic disease was detected in any patient with stage 0 or I disease the theoretical cost of detecting a metastasis was infinity. The cost of detecting one true-positive in patients with stage II-i disease ( $£ 200393$ by standard staging) is substantially higher than for stage II-ii patients ( $£ 8492)$; thereafter, the cost of detecting a metastasis continues to fall with increasing stage. It can also be seen that there is an overall cost saving if CT is used to replace the standard combination of CXR, BS and US.

\section{DISCUSSION}

We assessed local practice for the radiological staging of women presenting with a new diagnosis of breast cancer, without symptoms of metastases, over a 9-year period. Overall, the yield for detecting metastases is low in such asymptomatic patients, with no occult metastases detected in any patient with stage 0 or I disease. Furthermore, only two patients with stage II-i disease had possible evidence of occult metastases. In fact, in patients with stage 0 to II-i such screening is likely to lead to a greater degree of false-positive results: 40 (out of the 2199 patients). The yield is improved in those presenting with a higher stage of disease: 40 out 
of 413 patients with stage II-ii, III, or IV disease had true-positive results ( $v s 46$ false positives). These results show the benefit of a risk-stratified staging protocol for early breast cancer but underline the importance of making inclusion criteria clear and less open to interpretation. In this way the majority of occult metastases can be detected with minimal false positives, incidental findings and unnecessary radiation exposure.

The local guidelines for our review were, in retrospect, nonspecific and open to interpretation. Although the inclusion of patients with T4 disease or any evidence of malignant lymphadenopathy is very clear, the inclusion of 'patients with more locally advanced disease' is open to interpretation. In fact both national and international guidelines on the baseline imaging of breast cancer tend to be either open to interpretation or lack consensus. NICE guidelines from 2002 recommend that patients with T4 tumours (stage III + ) should have staging investigations, their 2009 update additionally accepts that there is currently insufficient evidence to support the choice of one imaging modality over another (NICE, 2002, 2009). The Scottish Intercollegiate Guidelines Network also states that only stage III + may require staging (SIGN, 2005). The National Comprehensive Cancer Network guidelines suggest that staging investigations are not required in asymptomatic stage I disease, nor many other patients with early stage breast cancer, and should only be considered in those with symptoms or T3, N1, M0 disease (stage IIIa) (NCCN, 2009). Conversely, the British Association of Surgical Oncology states that no asymptomatic patients should be staged, aside from pre-operative CXR (BASO, 2005).

These varying recommendations for imaging staging for occult metastases in women with newly diagnosed breast cancer reflect the paucity of evidence on this issue in the setting of early stage disease without symptoms suggestive of metastases. Internationally, the most robust guidelines seem to be those proposed by the Cancer Care Ontario Practice Guidelines Initiative (CCOPGI) (Myers et al, 2006). These guidelines are also endorsed by the National Guideline Clearinghouse - an initiative of the Agency for Healthcare Research and Quality which is affiliated with the American Medical Association and America's Health Insurance Plans. The guidelines are based on a literature search of peerreviewed articles on the baseline investigation of asymptomatic breast cancer patients up to 2003. They recommend no investigations for stage 0 and I patients, a BS for stage II disease and CXR, US and BS for stage III tumours.

Having decided which patients to investigate, it is then important to consider which modality or modalities are most appropriate for radiological staging. The trend we have observed locally is to move away from the more traditional methods of investigation with CXR, US and BS, to use CT of the chest, abdomen and pelvis. Certainly the CT option is logistically easier for the patient - it involves one visit to the department and the images can be acquired in a matter of seconds. If patients are required to have a CXR, US and BS, this will often involve separate attendances (often on separate days) to different areas of the hospital and, in the case of bone scan, returning $4 \mathrm{~h}$ after initial injection of isotope to complete the study. Indeed, a study into patient preference comparing CT with BS revealed that $75 \%$ patients preferred their experience of CT, compared with only $5 \%$ at BS (20\% no preference) (Groves et al, 2006). There is no question that CT chest is more sensitive and specific than CXR for diagnosing lung metastases, nevertheless, it does provide an increased radiation burden. Overall CT probably has a higher sensitivity than US for liver lesion detection. However, US will retain a role in targeting the biopsy of indeterminate lesions, or evaluation of lesions too small to characterise by CT.

Perhaps more controversial is the use of CT instead of BS. Computed tomography advances enable improved detection of bone metastases and there is evidence that this is equivalent to BS, with some authors concluding that the latter may be omitted (Groves et al, 2006; Bristow et al, 2008). Bone scintigraphy covers the entire skeleton (including long bones, neck and skull), however, of the 52 true-positive bone scan results in our study all had at least one metastasis within a field that would be covered by CT chest/abdomen/pelvis. Furthermore, detection of bone metastases at an asymptomatic stage has not been shown to prolong survival (Sato et al, 2003). Another issue is the increased radiation dose of CT. The effective dose of CT of the chest, abdomen and pelvis is $9.9 \mathrm{mSv}$ (Shrimpton et al, 2006), in comparison with a bone scan (740 MBq injected) at $4.2 \mathrm{mSv}$ (ICRP, 1988), and CXR at $0.02 \mathrm{mSv}$ (HPA, 2005). The long-term effects of radiation exposure are a concern; however, this is likely to be less relevant in patients with higher-stage breast cancer.

Out of 411 bone scans, 298 were negative, 52 were true positives and 61 were false positives. These results are similar to findings of Puglisi et al (2005) where 26 true-positive and 25 false-positive results were reported in 412 breast cancer patients of all stages. The false-positive rate of bone scans $(13.7 \%)$ is much higher than that of CT $(3.8 \%)$ in our study. The increased specificity of CT may reduce follow-up investigations, which could partially offset the increased radiation dose, limit the psychological burden of falsepositive results and reduce the need for further invasive testing. A meta-analysis of bone scan results in asymptomatic breast cancer patients by the CCOPGI group revealed differences in studies performed before 1980, with high pick-up rates, and those after, with a low rate of metastatic detection (Myers et al, 2006). The differences may be explained by differing thresholds for reporting positive results. In current practice, any equivocal bone scan result will lead to further imaging and possibly biopsy for confirmation of malignancy; thus in the earlier studies it is possible that some positive results were in reality false-positive results. Also the advent of screening programmes is expected to alter the demographics of the staging groups, with a higher proportion of patients presenting with earlier stage disease. Similar factors may help explain the relatively small incidence of metastases observed in our patient population, with low-stage disease: $0 \%$ of patients in stages 0 and I and only $0.3 \%$ of patients in stage II shown to be true positives.

This study was not designed for direct comparison of test accuracy, however, the apparent advantages of CT in relation to improved sensitivity and patient convenience, along with the local trend towards this form of imaging, have led us to recommend this as the baseline imaging modality of choice in patients presenting to our unit with asymptomatic newly diagnosed breast cancer. Given the relative lack of evidence directly comparing CT and BS for bone metastases in breast cancer, we are extending the CT protocol in breast cancer patients to include the supraclavicular fossa and proximal femur. The cost estimates in Table 4 are included to give an indication of the relative costs of implementing different imaging strategies based upon disease stage. Regardless of which stage of disease is used as a cut-off for imaging, our data suggest that implementing a strategy of using CT rather than conventional staging investigations (CXR, BS and US) would result in health-care cost saving.

\section{CONCLUSION}

The result of this study together with published evidence and existing guidelines has led us to adopt the following policy for the imaging of asymptomatic patients with newly diagnosed breast cancer, which we hope will maximise metastatic detection while minimising harmful side effects:

- Pre-operative CXR is not necessary as a staging investigation follow RCR guidelines (RCR, 2007)

- Patients with clinical stages III and IV disease (Greene et al, 2002) should undergo staging with CT of the chest (to include 
the supraclavicular fossa), abdomen and pelvis (including proximal femur). This includes patients with tumours that are:

- Larger than $5 \mathrm{~cm}$ and involve at least one axillary lymph node

- Any size and involve $\geqslant 4$ axillary lymph nodes

- Any size and involve at least one internal mammary, infraclavicular or supraclavicular lymph node

- Any size and involve the skin or chest wall (includes inflammatory breast cancer)

- In patients with stage III or IV disease where the results will not affect management, staging investigations are not required

\section{ACKNOWLEDGEMENTS}

Professor Gordon Wishart and Dr Peter Britton were supported with research funding from the NIHR Cambridge Biomedical Research Centre. We thank Emily Hind, Breast Cancer Trials Nurse for her help with some of the data collection.

Supplementary Information accompanies the paper on British Journal of Cancer website (http://www.nature.com/bjc)

\section{REFERENCES}

Avril N, Mather SJ, Roylance R (2007) FDG-PET and PET/CT in breast cancer staging. Breast Care 2: $372-377$

Bristow AR, Agrawal A, Evans AJ, Burrell HC, Cornford EJ, James JJ, Hamilton L, Robertson JF, Chan SY, Lawton PA, Cheung KL (2008) Can computerised tomography replace bone scintigraphy in detecting bone metastases from breast cancer? A prospective study. Breast 17(1): $98-103$

The Association of Breast Surgery at BASO, Royal College of Surgeons (2005) British Association of Surgical Oncology (BASO) guidelines for the management of symptomatic breast disease. Eur J Surg Oncol 31: S1 - S21.4

Fleming ID, Cooper JS, Henson DE, Hutter RV, Kennedy BJ, Murphy GP, O’Sullivan B, Sobin LH, Yarbro J (1997) AJCC Cancer Staging Manual, 5th edn. Lippincott-Raven: Philadelphia

Greene FL, Page DL, Fleming ID, Fritz AG, Balch CM, Haller DG, Morrow M (2002) AJCC Cancer Staging Manual. 6th edn Springer-Verlag: New York

Groves AM, Beadsmoore CJ, Cheow HK, Balan KK, Courtney HM, Kaptoge S, Win T, Harish S, Bearcroft PW, Dixon AK (2006) Can 16-detector multislice CT exclude skeletal lesions during tumour staging? Implications for the cancer patient. Eur Radiol 16(5): 1066-1073

Health Protection Agency (2005) Radiation Protection Division (formerly NRPB) Recommendations. HPA: England www.hpa.org.uk/radiation

International Commission on Radiological Protection (1988) ICRP Publication 53: Radiation Dose to Patients from Radiopharmaceuticals Annals of the ICRP Volume 18/1-4. IRCP: England

Myers R, Minuk T, Johnston M (2006) Diagnostic Imaging Guidelines Panel. Diagnostic Imaging in Breast Cancer: Recommendations Report. Cancer Care Ontario (CCO): Toronto www.cancercare.on.ca

National Comprehensive Cancer Network Breast Cancer Guideline, Version 1.2009 (2009), http://www.nccn.org

National Institute for Health and Clinical Excellence (NICE) (2002) Advanced breast cancer - diagnosis and treatment. 8 August 2002 http://www.nice.org.uk

National Institute for Health and Clinical Excellence (NICE) (2009) Advanced breast cancer - diagnosis and treatment. Clinical guideline 81. http://www.nice.org.uk
Office for National Statistics (2008) Cancer Statistics Registrations: Registrations of Cancer Diagnosed in 2005, England Series MB1 No.36. National Statistics: London www.statistics.gov.uk

Patanaphan V, Salazar OM, Risco R (1988) Breast cancer: metastatic patterns and their prognosis. South Med J 81(9): 1109-1112

Puglisi F, Follador A, Minisini AM, Cardellino GG, Russo S, Andreetta C, Di Terlizzi S, Piga A (2005) Baseline staging tests after a new diagnosis of breast cancer: further evidence of their limited indications. Ann Oncol 16(2): $263-266$

Puglisi F, Andreetta C, Fasola G, Cattaruzzi E, Geatti O (2007) Bone scan for baseline staging in invasive breast cancer at the time of primary presentation. Breast Care 2: 358-364

Ravaioli A, Passini G, Polselli A, Papi M, Tassinari D, Arcangeli V, Milandri C, Amadori D, Bravi M, Rossi D, Fattori PP, Pasquini E, Panzini I (2002) Staging of breast cancer: new recommended standard procedure. Breast Cancer Res Treat 72: $53-60$

Royal College of Radiologists (2006) Recommendations for Cross-Sectional Imaging in Cancer Management: Computed Tomography - CT, Magnetic Resonance Imaging - MRI, and Positron Emission Tomography - PET-CT (2006). Issue 2, pp 124-126. The Royal College of Radiologists: London, www.rcr.ac.uk

Royal College of Radiologists (2007) Making the Best Use of Clinical Radiology Services. 6th edn The Royal College of Radiologists: London www.rcr.ac.uk

Sato N, Hino M, Sano M (2003) Detection of bone metastases in routine follow-up after treatment for primary breast cancer. Breast Cancer 10(4): $335-340$

Scottish Intercollegiate Guidelines Network (SIGN), Publication 84 (2005) Management of breast cancer in women - a national clinical guideline. www.sign.ac.uk/pdf/qrg84.pdf

Shrimpton PC, Hillier MC, Lewis MA, Dunn M (2006) National survey of doses from CT in the UK: 2003. Br J Radiol 79: 968-980

World Health Organization International Agency for Research on Cancer (2003) World Cancer Report. www.who.int

World Health Organization (2006) Fact sheet no 297: cancer. www.who.int 